\title{
A new formalism for representation of heat capacities of metals
}

\author{
J P HAJRA \\ Department of Metallurgy, Indian Institute of Science, Bangalore 560012 , India \\ MS received I April 1987; revised 10 June 1987
}

\begin{abstract}
The applicability of a function involving geometrical progression of temperature in interpreting the heat capacities of metals has been studied. The constants of the function have been described in terms of vibrational, electronic and magnetic contributions to heat capacities. The equation may he useful in representing heat capacity of metals.
\end{abstract}

Keywords. Vibrational constant; magnetic constant; electronic heat capacities.

\section{Introduction}

Various empirical mathematical equations are used for presentation of heat capacities of metals as a function of temperature. Although extrapolation of heat capacities with respect of temperature sometimes leads to difficulties, the equations are important in determining the thermodynamic properties of metals.

\section{Formalisms consisting of two and three parameters}

Heat capacities of solid metals and intermetallic compounds at elevated temperature are frequently expressed by a linear form of function

$$
C_{p}=A+B T,
$$

where $A$ and $B$ are constants and evaluated by least mean squares regressional analysis of the experimental values over a specified range of temperature. The choice of an equation depends on the temperature range and the way in which the slope of the $C_{p}$ curve varies. Sometimes, heat capacity shows pronounced curvature at low temperature and becomes linear at high temperature. In such cases, a threeparameter form of equation,

$$
C_{p}=A^{\prime}+B^{\prime} T+C^{\prime} T^{-2}
$$

is generally preferred as proposed by Kelley (1960). The equations are purely empirical in nature and limited to a range of temperature of the data from which they have been derived. Apart from (1) and (2), an additional term of the form $d^{\prime} T^{2}$ or $d T^{1 / 2}$ has also been incorporated by Kubaschawski et al (1979) in deriving heat capacities of various metals and inorganic substances. Battezzati and Garrone (1984) recently observed that the $\Delta C_{p}$ between the undercooled melt and solid equilibrium phases of metallic glass forming alloys decreases with increase in temperature. The variation of the $C_{p}$ data as a function of temperature is considered by them to be either linear or intermediate between linear and hyperbolic. The hyperbolic type of representation is denoted by

$$
C_{p}=x+y / T
$$

where $x$ and $y$ are constants. 


\subsection{The present equation}

The dependence of heat capacity on temperature is usually expressible in the form of polynominals, as already mentioned, and the corresponding coefficients which are important in describing the behaviour as a function of temperature. The present study investigates the form of function

$$
C_{p}=a /(1+b T),
$$

where $a$ and $b$ are constants and are to be evaluated for a particular system under consideration.

\section{Results and discussion}

The applicability of the equation has been studied carefully choosing the selected $C_{p}$ values of several metals from Hultgren et al (1973). For comparison, the constants involved in (1), (2) and (3) have also been evaluated using least means squares regressional analysis of the experimental values computed by Hultgren et al (1973). The evaluated constants for (1), (2), (3) and (4) are computed in table 1 along with their respective standard deviations. The magnitude of the standard deviations for metals, such as $\mathrm{Ag}, \mathrm{Au}, \beta \mathrm{Co}, \mathrm{Cu}, \mathrm{Ni}, \mathrm{Zn}$ and $\mathrm{Cr}$ at the stated temperature range, show that the data derived by the present equation are in good agreement with those of the experimental values of Hultgren et al (1973). The hyperbolic function shows consistently higher standard deviations for all the metals than those derived by (1), (2) and (4). Although comparable deviations from experimental values have been registered by (1), (2) and (4) for $x$. Co and $\mathrm{Pt}$, the present equation exhibits somewhat higher values for $x \mathrm{Mn}$ than those derived by (1) and (2). The deviations, however, are generally considered to lie within the range of experimental error. Figure 1 presents the comparison of $C_{p}$ values for some of the metals which are derived by (1), (2) and (4) and the experimental data reported by Hultgren et al (1973).

\section{Physical interpretation and form of the present equation}

The constants $a$ and $b$ of the expanded form of the present equation may be interpreted by considering the vibrational, electronic and magnetic contributions to heat capacity. Equation (4) may be expanded in power series of $T$ as

$$
C_{p}=a /(1+b T)=a(1-b T)
$$

The empirical approach by Weiss and Tauer (1956) and Hofmann et al (1956) is to split up the experimental specific heat data in additive vibrational, electronic and magnetic contributions. The vibrational contribution to the specific heat is calculated from Debye model as described by Kittel (1976) as

$$
C_{v}=9 R(1 / x)^{3} \int_{0}^{x} \frac{x^{4} \cdot e^{x}}{\left(e^{x}-1\right)^{2}} \mathrm{~d} x,
$$

where $x=\theta / T$ and $\theta$ is the Debye temperature. A good description of $C_{v}$ can be obtained if $\theta$ is known accurately. The Debye temperature $\theta$, has however been 


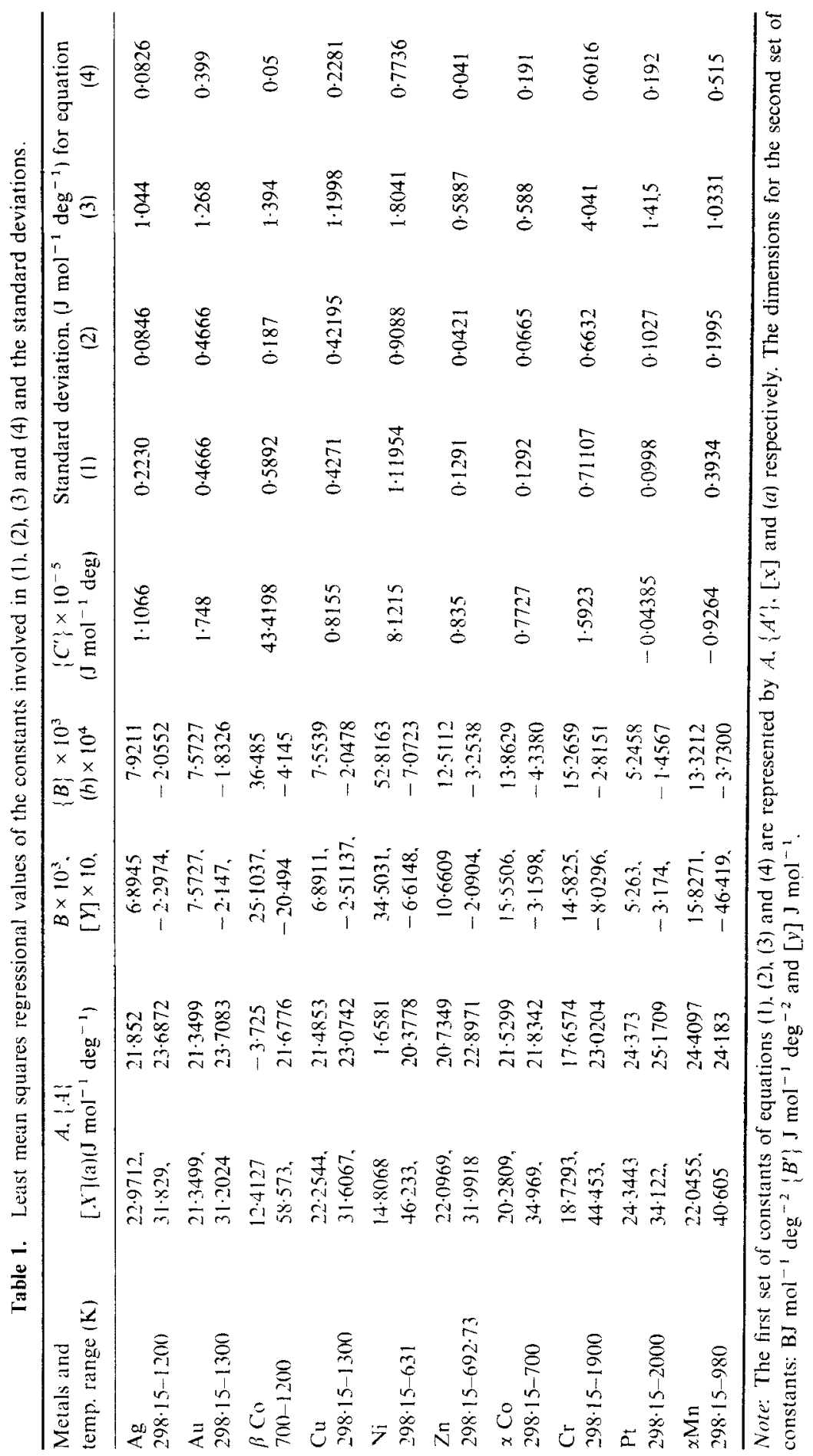




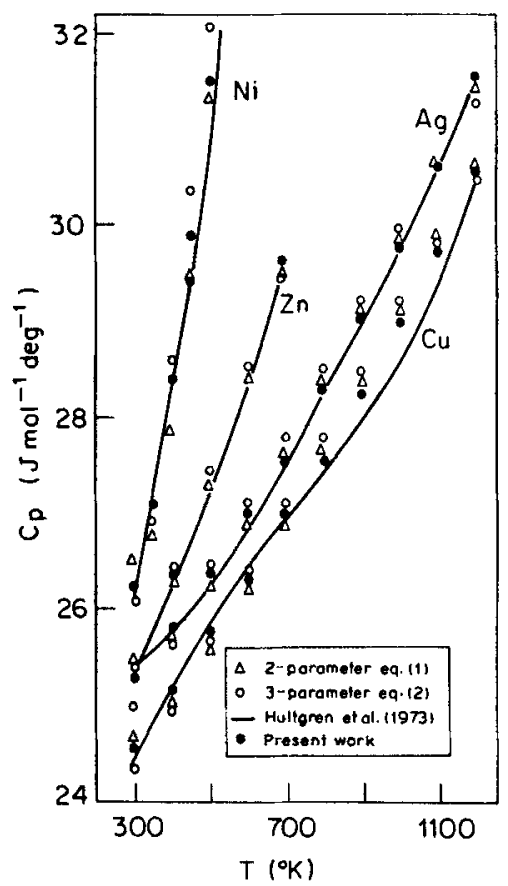

Figure 1. Comparison of heat capacities of metals between the experimental values and those derived from the models.

shown by Desorbo (1954) to vary with experimental temperature for many metals due to lattice irregularities and crystal structure. The variation of $\theta$ with temperature has further been found by Desorbo (1954) to be significant between 0 and $20 \mathrm{~K}$ and almost linear with slight positive slope in the neighbourhood of $100 \mathrm{~K}$. Because of this variation and in the absence of any other experimental data, $\theta$ for various metals has been selected as shown in table 2 . To calculate $C_{v}$, the temperature is assumed to be $298.15 \mathrm{~K}$, as the $C_{p}$ data for various metals are quoted from that temperature. Table 2 shows that a reasonable agreement exists between the Debye values at $298.15 \mathrm{~K}$ and $a$ values of the present equation. The latter values are considered to be closer to the Debye values than those derived by (1), (2) and (3).

The correction due to lattice dilation, $\left(C_{p}-C_{v}\right)$ is small compared to $C_{v}$ and the following empirical equation as suggested by Kaufman et al (1963) is used as an approximation over the entire range of temperature as

$$
C_{d}=10^{-4} C_{v} T
$$

Table 2. Comparison between $a$ values of (4) and Debye heat capacities $\mathrm{Cv}$ at $298 \cdot 15 \mathrm{~K}$.

\begin{tabular}{|c|c|c|c|c|c|c|c|c|c|}
\hline Metals & $\mathrm{Ag}$ & $\overline{\mathrm{Au}}$ & $\operatorname{Co}(\alpha)$ & $\mathrm{Cu}$ & $\mathrm{Ni}$ & $\mathrm{Zn}$ & $\mathrm{Cr}$ & $\mathrm{Pt}$ & $\mathrm{Mn}$ \\
\hline $\begin{array}{l}\text { Debye temp. } \theta,(\mathrm{K}) \\
\text { Cv } 298 \cdot 15 \mathrm{~K}\end{array}$ & 225 & 164 & 445 & 339 & 456 & 310 & 402 & 229 & 450 \\
\hline $\begin{array}{l}\left(\mathrm{J} \mathrm{mol}^{-1} \mathrm{deg}^{-1}\right) \\
a \text { equation }(4)\end{array}$ & $24 \cdot 26$ & 24.57 & $22 \cdot 32$ & $23 \cdot 38$ & $22 \cdot 13$ & 23.63 & 22.698 & $24 \cdot 195$ & $21 \cdot 2$ \\
\hline$\left(\mathrm{J} \mathrm{mol}^{-1} \mathrm{deg}^{-1}\right)$ & 23.687 & 23.708 & $21 \cdot 834$ & $23 \cdot 074$ & $20 \cdot 377$ & $22 \cdot 89$ & 23.02 & $25 \cdot 17$ & $24 \cdot 18$ \\
\hline
\end{tabular}


Table 3. Comparison between $a b$ values of (5) with the lattice dilation and electronic specific heat values of metals.

\begin{tabular}{lcc}
\hline Metals & $\begin{array}{c}\left(\gamma_{e}+C_{b}\right) \times 10^{4}, \\
\left(\mathrm{~J} \mathrm{~mol}^{-1} \mathrm{deg}^{-2}\right)\end{array}$ & $\begin{array}{c}a b \times 10^{4}, \\
\left(\mathrm{~J} \mathrm{~mol}^{-1} \mathrm{deg}^{-2}\right)\end{array}$ \\
\hline $\mathrm{Ag}$ & 30.53 & 48.68 \\
$\mathrm{Au}$ & 31.85 & 43.44 \\
$\mathrm{Co}(\alpha)$ & 69.77 & 94.71 \\
$\mathrm{Cu}$ & 30.33 & 47.25 \\
$\mathrm{Ni}$ & 92.42 & 144.11 \\
$\mathrm{Cr}$ & 38.6 & 64.80 \\
$\mathrm{Pt}$ & 90.42 & 36.6 \\
$\mathrm{Mn}$ & 147.7 & 90.20 \\
\hline
\end{tabular}

The other coefficient of $T$ from theoretical consideration is the electronic specific heat, $\gamma_{e}$, and expressed from the free electron model (6) as described by Hofmann et al (1956) as

$$
C_{e}=\gamma_{e} T \text {. }
$$

The latter values for various metals are obtained from Hultgren et al (1973). The coefficients of $T,\left(10^{-4} C_{v}+\gamma_{e}\right)$ from (7) and (8) are compared with $a b$ terms of the expanded function (5) in table 3 . The two sets of data compare favourably despite various uncertainties. Since low temperature electronic heat values are used, uncertainties lie the way they may vary with temperature. Although the $C_{v}$ values have been calculated from Debye function (equation (6)) at $298.15 \mathrm{~K}$ to be used in (7), the overall lattice dilation values will not change significantly with increase in temperature relative to $298 \cdot 15 \mathrm{~K}$. Table 3 also shows that the $a b$ values of the present equation for various metals are closer to the $\left(10^{-4} C_{v}+\gamma_{e}\right)$ values than the

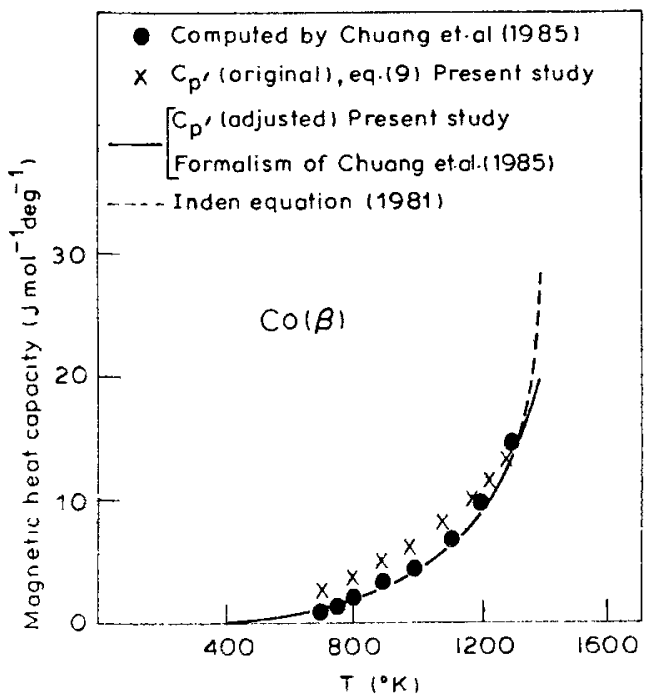

Figure 2. Magnctic specific heat for $\beta$ cobalt. Comparison between the derived values and those from the experiment. 


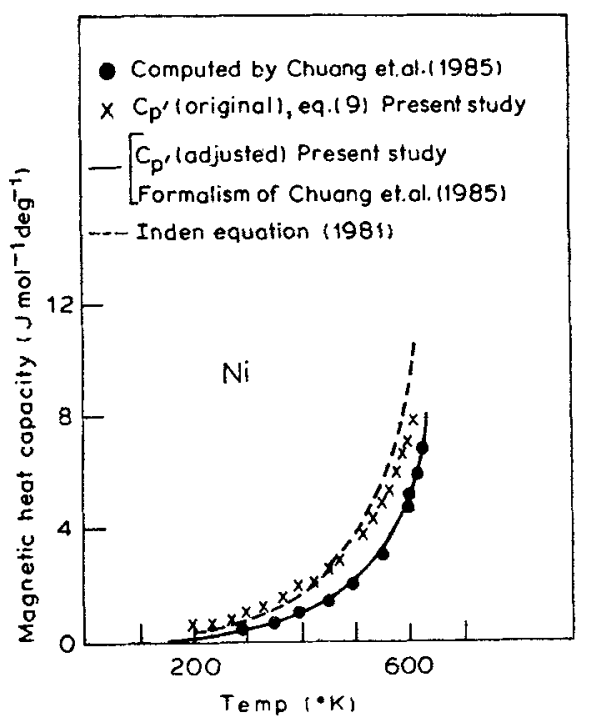

Figure 3. Magnetic specific heat for nickel. Comparison between the derived values and those from the experiment.

corresponding coefficients of $T$ of (1) and (2). The rest of the present function may be expressed as

$$
C_{p}^{\prime}=[a /(1+b T)]-a(1-b T)=a b^{2} T^{2} /(1+b T) .
$$

The applicability of the equation has been studied carefully choosing the selected values of the magnetic specific heats of $\mathrm{Co}(\beta)$ and Ni computed by Chuang et al (1985) below the curie temperature. The equation records somewhat higher values than the experimental data as shown in figures 2 and 3. The figures show that the magnetic contribution to heat capacity cannot be calculated directly by using (9). This is considered to be due to the increased vibrational effects which is difficult to separate from the magnetic contributions. It may be noted that at $298.15 \mathrm{~K}$, the Debye values of various metals do not exceed the value of $3 R$ which corresponds to the maximum frequency of vibration of atoms $v_{m}$. Since $C_{v}$ increases with increase in temperature, the coefficients of higher powers of $T$ of the expanded function (5) are expected to be associated with atomic vibration. To study the suitability of the function (9), regressional analysis of the magnetic heat capacities of $\mathrm{Co}(\beta)$ and $\mathrm{Ni}$ has been carried out. The least mean squares regressional values of $a$ and $b$ along with the standard deviations are computed as follows:

\begin{tabular}{lccc}
\hline & $\begin{array}{c}a \\
\left(\mathrm{~J} \mathrm{~mol}^{-1} \mathrm{deg}^{-1}\right)\end{array}$ & $b \times 10^{4}$ & $\begin{array}{c}\text { Standard deviation } \\
\left(\mathrm{J} \mathrm{mol}^{-1} \mathrm{deg}^{-1}\right)\end{array}$ \\
\hline $\mathrm{Co}(\beta)$ & 3.968 & -6.35 & 0.035 \\
$\mathrm{Ni}$ & 1.6883 & -13.33 & 0.024 \\
\hline
\end{tabular}

The adjusted $C_{p}$ (magnetic) values as derived by (9) are in good agreement with those of the experimental values as shown in figures 2 and 3 . Since the equation with 
different sets of constants interprets the magnetic properties of the metals, the form of the function is considered to be adequate below the curie temperature. The magnetic transition, however, is described by Belov (1961) as a second-order transformation and $C_{p}$ at curie temperature being a discontinuous function the present equation cannot be extrapolated beyond the curie temperature. It may be mentioned that the regressional analysis of the experimental $C_{p}$ data for $\operatorname{Co}(\beta)$ and $\mathrm{Ni}$ has been carried out as reported in table 1 , in the temperature range of 700 to $1200 \mathrm{~K}$ and $298.15 \mathrm{~K}$ to $631 \mathrm{~K}$ respectively. The applicability of the present equation is, therefore, restricted to the temperature range where the data have been fitted. Empirical mathematical equations consisting of adjustable parameters have been proposed by Chuang et al (1985) and Inden (1981) for the magnetic contribution to the specific heats of pure metals. The derived values from the formalisms are also shown in figures 2 and 3 for $\mathrm{Co}(\beta)$ and $\mathrm{Ni}$ respectively; the values are found to coincide with the adjusted $C_{p}$ (magnetic) values from (9).

Table 1 further indicates that the present equation records lower values of standard deviations than those derived by the conventional two- and threeparameter equations. Since the applicability of the equation spans over a fairly wide temperature range, the equation is considered to be useful in interpolation of heat capacity values and evaluation of various thermodynamic functions of the metals.

\section{Conclusions}

The present equation offers an alternative expression to two- and three-parameter functions for representation of heat capacities of metals. The constants of the equation are susceptible to physical interpretation based on vibrational, electronic and magnetic contributions to heat capacity.

\section{References}

Battezzati L and Garrone E 1984 Z. Metallk. 75305

Belov K P 1961 Magnetic transitions (New York: Consultants Bureau) p. 1

Chuang Y Y, Schmid R and Chang Y A 1985 Metall. Trans. 16153

Desorbo W 1954 Acta Metall. 2274

Hofmann J A. Paskin A, Tauer K J and Weiss R J 1956 J. Phys. Chem. Solids 145

Hultgren R, Desai P D, Hawkins D T, Gleiser M, Kelly K K and Wagman D P 1973 Selected values of the thermodynamic properties of the elements (Ohio: ASM)

Inden G 1981 Physica B103 82

Kaufman L, Clougherty E V and Weiss R J 1963 Acta Metall. 11323

Kelly K K 1960 Contribution to the data on theoretical metallurgy U. S. Bur. Mines Bull. p. 584

Kittel C 1976 Introduction to solid state physics (New York: John Wiley) 5th ed.

Kubaschewski O and Alcock C B 1979 Metal thermochem (Oxford: Pergamon Press) 5th ed.

Weiss R J and Tauer K J 1956 Phys. Rev. 1021490 